\title{
Nota sobre 0 uso de bio-sensores "Maria" nas ações de vigilância entomológica contra a doença de Chagas ao norte de Minas Gerais
}

\author{
About the use of "Maria" bio-sensors in the activities of epidemiological \\ surveillance against Chagas' disease in the north \\ of Minas Gerais State
}

\author{
João Carlos Pinto Dias ${ }^{1}$, Edvá de Paula Vieira ${ }^{2}$, Hélio Tadashi ${ }^{2}$ \\ e Bernardino Vaz de Mello Azeredo ${ }^{3}$
}

\begin{abstract}
RESUMO
Oitocentos e sessenta e seis bio-sensores para a detecção passiva de triatomíneos foram ensaiados em intradomicílios de treze municípios de área endêmica de Triatoma sordida (Norte de Minas Gerais, Brasil), espécie que é hoje a mais freqüentemente detectável no Brasil, especialmente naquela Região. Examinados os sensores a cada três meses, por quatro vezes em uma subárea de sete municípios ( seiscentos e quarenta e dois sensores, com positividade máxima de $0,5 \%$ ) e por duas nos outros seis municípios (duzentos e vinte e quatro sensores, com positividade máxima de 2,7\%), os resultados foram, significativamente inferiores à rotina de busca direta hora-homem feita nos mesmos municípios, inclusive para as taxas de infestação intradomiciliar. Em que pese a simplicidade e boa aceitação dos sensores pela população, os mesmos não se mostraram adequados à pesquisa triatomínica na região em apreço, tanto em termos de efetividade quanto de custo-benefício.
\end{abstract}

Palavras-chaves: Doença de Chagas. Controle. Bio-sensores.

\begin{abstract}
Eight hundred and sixty six bio-sensors for the passive detection of triatomine bugs, were tried in intra-domiciles of thirteen municipalities of an endemic area of Triatoma sordida in the North of Minas Gerais State, Brazil. This species is nowadays the most captured in Brazil and mainly in the above region. The sensors were examined every three months, corresponding to four times in the first seven municipalities ( six hundred and forty two sensors, with a maximum positivity of $0.5 \%$ ) and two times in the other six (two hundred and twenty two sensors, with a maximum positivity of $2.7 \%$ ), showing results significantly inferior in comparison with the routine man- hour research performed in the same municipalities, including in terms of intra-domestic infestation index. Despite the simplicity and good acceptance of the sensors by the population they were demonstrated to be inadequate for triatomine research under the conditions of this trial, in terms of efficacy and cost-benefit.
\end{abstract}

Key-words: Chagas' disease. Control. Bio-sensors.

No contexto da luta contra a doença de Chagas, particularmente no Brasil, um dos desafios do presente momento corresponde à detecção e eliminação de focos residuais ou adventícios de triatomíneos na etapa de vigilância epidemiológica, mormente quando em jogo baixas densidades do inseto ${ }^{9} 10^{15}{ }^{37}$. Em tal situação, que abarca a maior parte da área endêmica do país, a captura desses vetores pela metodologia que a Fundação Nacional de Saúde (FUNASA) empregava para a fase de ataque (busca ativa direta por agentes federais) é onerosa e lenta, preferindo-se a estratégia de detecção passiva pela própria população, mediante participação comunitária estimulada por contínua educação sanitária ${ }^{67} 3234$. Entre as

1. Centro de Pesquisas René Rachou da Fundação Oswaldo Cruz, Belo Horizonte, MG. 2. Setor Técnico da Fundação Nacional de Saúde de Minas Gerais, Belo Horizonte, MG. 3. Gerência de Doença de Chagas da Secretaria de Estado da Saúde, Belo Horizonte, MG.

Endereço para correspondência: Dr. João Carlos Pinto Dias. Centro de Pesquisas René Rachou/FIOCRUZ. Av. Augusto de Lima 1715, 30190-002 Belo Horizonte, MG, Brasil.

e-mail: jcpdias@ cpqrr.fiocruz.br

Recebido para publicação em 26/2/2004

Aceito em 18/6/2005 
possibilidades de detecção continuada de focos, há tempos foi muito estimulado pelo TDR/OMS o uso de bio-sensores para triatomíneos no ambiente domiciliar, desenvolvidos na década de 1960, na Venezuela( "caixa de Gómez-Nuñez" ), e nos anos 1980 na Argentina ( com o nomede "bio-sensores Maria) ${ }^{17}{ }^{213135}$. Colocadas em pontos estratégicos do intradomicílio, estas caixas oferecem abrigo a eventuais triatomíneos ali arribados ou existentes, que nelas se escondem e mesmo nidificam, facilitando sua detecção e captura por agentes de saúde e pela comunidade ${ }^{3135}$.

A experiência maior com estes bio-sensores tem ocorrido na Argentina, no trabalho de consolidação do controle do Triatoma infestans, cujos focos são ali primordialmente intradomiciliares ${ }^{41}$. Pequeno ensaio para a mesma espécie com método semelhante, já fora realizado no Brasil por Forattini, Garcia Zapata, e Pinchin cols, seguindo o trabalho pioneiro de Gómez Nuñes, no controle de Rhodnius prolixus na Venezuela, em $19655^{1315171825}$.

Objetivando ampliar a efetividade das ações de vigilância entomológica no controle da doença de Chagas no Brasil, ao final dos anos 1990 testou-se em alguns municípios do norte de Minas Gerais o bio-sensor "Maria" de procedência argentina, gentilmente cedido pela Dra Elza L. Segura, então diretora do Instituto Malbrán, de Buenos Aires, a quem agradecemos.

\section{MATERIAL E MÉTODOS}

A área do ensaio correspondeu a treze municípios do Norte de Minas Gerais, em fase de consolidação da eliminação de T. infestans, no âmbito da "Iniciativa do Cone Sul" 1011 37. Nesta região, as ações de profilaxia anti vetorial da Fundação Nacional de Saúde se intensificaram na segunda metade dos anos de 1990, logrando-se a certificação desta eliminação no Estado por comissão externa da OPS/OMS, em $2001^{711}$. Por razões administrativas, estabeleceram-se duas subáreas, para acompanhamento dos bio-sensores por duas diferentes equipes de agentes de saúde: um grupo de sete municípios mais ao norte da macro-região (Icaraí de Minas, Itacarambi, Manga, Januária, Miravânia, São Francisco e Montalvânia), aqui denominado "subárea A", e um grupo de seis municípios a nor-nordeste ( Fruta de Leite, Gameleira, Riacho dos Machados, Rio Pardo de Minas, São João da Ponte e Varzelândia), denominado "subárea B". São municípios pobres e pequenos, com histórico recente de T. infestans, típicos do "Norte de Minas" e que se encontravam sob ações intensificadas de controle na época (1997-98), sendo submetidos a pesquisa triatomínica integral ( visita e busca ativa por agentes da FUNASA em todas as unidades domiciliares existentes) pela metodologia tradicional do órgã $0^{81433}$.

Os bio-sensores utilizados foram os de modelo "Maria", desenvolvidos na Argentina a partir da "caixa de Gomez-Nuñes" ${ }^{18} 35$. Correspondem a caixas simples de papelão, na medida aproximada de $30 \times 40 \times 7 \mathrm{~cm}$, com fundo falso e interior revestido por papelão fino sanfonado, com orifícios de passagem, muito adequadas como abrigo de triatomíneos ${ }^{42} 3135$. Sobre a tampa externa são coladas figuras coloridas de vários motivos, com objetivos estéticos e de aceitação pelos moradore ${ }^{35}$. Face ao limitado número de bio-sensores disponíveis (novecentos) e ao total de unidades domiciliares existentes nos treze municípios (78.442), optou-se por sortear para cada município, em quadrantes opostos, 2,5\% das localidades existentes, com captura anterior de T. infestans, para nestas colocar os biosensores em casas aleatoriamente selecionadas.

Foi estimado um total de 300 casas com bio-sensores, sendo 225 casas na subárea A, com 675 bio-sensores e 75 casas na subárea B (menos povoada, perfazendo 225 caixas), à razão geral de 3 bio-sensores por casa. Programaram-se três biosensores por casa, ou seja, um por quarto de dormir ( colocandose um na sala quando havia somente dois quartos na habitação) , à meia altura da parede, conforme a experiência argentina ${ }^{435}$. Năo foram colocados bio-sensores em anexos peridomiciliares.

A colocação e revisão periódica das caixas foi feita por pessoal da FUNASA engajado no programa de doença de Chagas e devidamente capacitado ao uso desta ferramenta, programando-se uma revisão de todos os bio-sensores a cada três meses, durante um ano. Na subárea "A" foram efetivamente instalados 642 bio-sensores e na "B" 224, conforme os critérios acima. Os triatomíneos detectados foram encaminhados para identificação no Laboratório Regional de Entomologia da FUNASA, então instalado no seu Distrito Sanitário de Montes Claros.

Outros artrópodes coletados foram apenas rotulados por seu grupo genérico pelos inspetores encarregados, que anotaram todo 0 movimento das revisões em planilha própria baseada nos modelos correntes da FUNASA ${ }^{14}$. A priori, convencionou-se não levar em conta eventuais vestígios representados por fezes depositadas no interior dos bio-sensores, para evitar problemas de especificidade neste ensaio, apenas registrando-se triatomíneos ( exemplares vivos ou mortos), exúvias e ovos. Por razões de extrema limitação de pessoal, na subárea "B" ocorreram apenas duas revisões. Por estas mesmas razões, 0 cotejo direto da eficácia dos bio-sensores com 0 método tradicional de pesquisa hora-homem não pôde ser realizado concomitantemente nas casas selecionadas, como se pretendia, optando-se por fazer uma comparação genérica dos dados obtidos pelos bio-sensores com aqueles do trabalho da normal da FUNASA, na mesma macro-região e em cada município, na mesma época.

Năo foram utilizados produtos desalojantes na pesquisa homem/ hora deste trabalho. Por razões éticas e técnicas, todas as unidades domiciliares encontradas positivas para triatomíneos foram expurgadas com piretróide, conforme a rotina da FUNASA ${ }^{74}$.

\section{RESULTADOS}

Dos 642 bio-sensores efetivamente instalados na subárea " $\mathrm{A}$ ", ao fim da observação de doze meses houve perda ou danificação de 14\%, dado não levantado na área "B". Estas perdas deveramse basicamente à falta de cuidados por parte da população, geralmente retirando as caixas e deixando-as no meio ambiente.

De modo geral, o poder de deteç̧ão de triatomíneos pelos bio-sensores na área estudada foi muito discreto e aleatório, observando-se pouca sensibilidade com relação aos indicadores 
tradicionais. Assim, por comparação genérica com os dados de 1997-1998 para 35 municípios da macro-região, as taxas máximas de dispersão (localidades positivas/localidades examinadas x 100) detectadas pelos bio-sensores foram de 13,04\%, na subárea "A", e de 38,46\%, na "B" (Tabelas 1 e 2), contra a taxa geral para toda a macro-região de $57,02 \%$, pela metodologia usual de busca homem-hora.

A variação desta taxa em doze Municípios deste estudo oscilou entre 48,2 e 100\% (Tabelas 3 e 4). Já as taxas de infestação ( casas positivas/casas examinadas x 100) detectadas pelos bio-sensores variaram de 0,0 a 1,3\% na subárea "A" e de 6,6 a $7 \%$ na "B", contra a taxa regional de $14,1 \%$ pela metodologia tradicional. Nas duas subáreas investigadas, as taxas mínimas e máximas de infestação intra e peridomiciliar foram respectivamente 0, 7 a 13\% e de 2,2 a 41,1\% (Tabelas 3 e 4). Já os índices de captura (densidade relativa $=$ № de triatomíneos capturados/№ de casas examinadas) foram respectivamente de 0,03 para 0 acumulado dos bio-sensores e 0,38 para as capturas manuais de rotina.

Os resultados mais discriminados dos bio-sensores por subregião " A" e "B" encontram-se respectivamente nas Tabelas 1 e 2, notando-se que apenas duas espécies de triatomíneos foram detectadas pelas caixas, correspondendo a quinze espécimes de Triatoma sordida (todos adultos (nove fêmeas) e um de T. pseudomaculata (adulto macho) . № presente trabalho não foram detectados ovos, ninfas ou exúvias nos bio-sensores, 0 que sugere não-colonização dos mesmos nesta experiência. Dejeções de triatomíneos não foram propositadamente

Tabela 1 - Resultados do emprego de 642 bio-sensores "Maria" para a detecção de triatomíneos em 225 casas de sete municípios (subárea "A") do norte de Minas Gerais, Brasil em 1997.

\begin{tabular}{|c|c|c|c|c|c|c|c|c|}
\hline \multirow[t]{2}{*}{ Mês } & \multirow{2}{*}{$\begin{array}{c}\text { Bio-sensores } \\
\text { examinados } \\
n^{0}\end{array}$} & \multicolumn{2}{|c|}{$\begin{array}{c}\text { Bio-sensores } \\
\text { positivos }\end{array}$} & \multirow{2}{*}{$\begin{array}{c}\text { Infestação } \\
\text { detectada } \\
\%\end{array}$} & \multirow{2}{*}{$\begin{array}{c}\text { Triatomíneos } \\
\text { detectados } \\
n^{0}\end{array}$} & \multirow[t]{2}{*}{$\begin{array}{c}\text { Espécies } \\
\text { detectadas* }\end{array}$} & \multicolumn{2}{|c|}{$\begin{array}{l}\text { Bio-sensores com } \\
\text { outros artrópodes }\end{array}$} \\
\hline & & $\overline{n^{0}}$ & $\%$ & & & & $\mathrm{n}^{0}$ & $\%$ \\
\hline Maio & 604 & 2 & 0,3 & 0,9 & 2 & T. sd & 64 & 10,6 \\
\hline Julho & 598 & 0 & 0,0 & 0,0 & 0 & - & 72 & 12,0 \\
\hline Setembro & 613 & 0 & 0,0 & 0,0 & 0 & - & 83 & 13,5 \\
\hline Novembro & 577 & 3 & 0,5 & 1,3 & 3 & T. sd, T. psm & 88 & 15,2 \\
\hline
\end{tabular}

*: T. sd = Triatoma sordida; $\mathrm{T}$. psm = Triatoma pseudomaculata

Tabela 2 - Resultados do emprego de 224 bio-sensores "Maria" para a deteccão de triatomíneos em 75 casas de seis municípios (subárea “ B”) do norte de Minas Gerais, Brasil, em 1997.

\begin{tabular}{|c|c|c|c|c|c|c|c|c|}
\hline \multirow[t]{2}{*}{ Mês } & \multirow{2}{*}{$\begin{array}{c}\text { Bio-sensores } \\
\text { examinados } \\
n^{0}\end{array}$} & \multicolumn{2}{|c|}{$\begin{array}{c}\text { Bio-sensores } \\
\text { positivos }\end{array}$} & \multirow{2}{*}{$\begin{array}{c}\text { Infestação } \\
\text { detectada } \\
\%\end{array}$} & \multirow{2}{*}{$\begin{array}{c}\text { Triatomíneos } \\
\text { detectados } \\
n^{0}\end{array}$} & \multirow[t]{2}{*}{$\begin{array}{c}\text { Espécies } \\
\text { detectadas* }\end{array}$} & \multicolumn{2}{|c|}{$\begin{array}{l}\text { Bio-sensores com } \\
\text { outros artrópodes }\end{array}$} \\
\hline & & $\overline{n^{0}}$ & $\%$ & & & & $\mathrm{n}^{0}$ & $\%$ \\
\hline Agosto & 171 & 4 & 2,3 & 7,0 & 5 & T. sordida & 19 & 11,1 \\
\hline Novembro & 183 & 5 & 2,7 & 6,5 & 5 & T. sordida & 23 & 12,6 \\
\hline
\end{tabular}

Tabela 3 - Índices de dispersão e infestação (\%) e espécies detectadas, da busca triatomínica pelo método de pesquisa direta (hora-homem) nos 7 municípios da subárea “ A” , em Minas Gerais, 1997.

\begin{tabular}{|c|c|c|c|c|c|c|c|}
\hline \multirow[b]{2}{*}{ Municípios } & \multirow{2}{*}{$\begin{array}{l}\text { Índice de } \\
\text { dispersão }\end{array}$} & \multicolumn{3}{|c|}{ Índice de infestação } & \multicolumn{3}{|c|}{ Espécies detectadas, por ordem } \\
\hline & & intra & peri & total & principal & segunda & terceira \\
\hline Icaraí de Minas* & 96,6 & 1,8 & 19,7 & 21,1 & T. sordida & T. pseudomaculata & - \\
\hline Itacarambi & 61,0 & 1,2 & 12,5 & 13,3 & T. sordida & T. pseudomaculata & P. diasi \\
\hline Januária* & 70,3 & 0,7 & 9,1 & 9,7 & T. sordida & R. neglectus & - \\
\hline Manga & 79,8 & 1,7 & 17,8 & 19,0 & T. sordida & R. neglectus & - \\
\hline Miravânia* & 94,3 & 2,2 & 17,3 & 19,0 & T. sordida & & - \\
\hline Montalvânia & 94,3 & 2,2 & 24,1 & 25,6 & T. sordida & P. diasi & - \\
\hline S. Francisco* & 92,3 & 3,8 & 27,9 & 30,3 & T. sordida & T. vitticeps & - \\
\hline
\end{tabular}

* - Neste município capturaram-se resíduos isolados de T. infestans em 1997, não mais detectados em anos seguintes ${ }^{11}$.

Tabela 4 - Índices de dispersão e infestação (\%) e espécies detectadas, da busca triatomínica pelo método de pesquisa direta (hora-homem) nos 6 municípios da subárea “ B” , em Minas Gerais, 1997.

\begin{tabular}{|c|c|c|c|c|c|c|c|}
\hline \multirow[b]{2}{*}{ Municípios } & \multirow{2}{*}{$\begin{array}{l}\text { Índice de } \\
\text { dispersão }\end{array}$} & \multicolumn{3}{|c|}{ Índice de infestação } & \multicolumn{3}{|c|}{ Espécies detectadas, por ordem } \\
\hline & & intra & peri & total & principal & segunda & terceira \\
\hline Gameleira & 96,7 & 13,0 & 28,2 & 35,6 & T. sordida & T. pseudomaculata & - \\
\hline R. Machados & 50,0 & 0,0 & 3,1 & 3,1 & T. sordida & - & - \\
\hline Rio P. de Minas & 48,3 & 0,5 & 2,2 & 2,7 & T. sordida & T. pseudomaculata & - \\
\hline S. João da Ponte & 100,0 & 8,0 & 41,1 & 45,3 & T. sordida & T. pseudomaculata & P. diasi \\
\hline Varzelândia & 93,9 & 4,2 & 33,2 & 35,3 & T. sordida & - & - \\
\hline Fruta de Leite & \multicolumn{7}{|c|}{ Não constam atividades de pesquisa direta em 1997} \\
\hline
\end{tabular}


contabilizadas, face à inexperiência dos agentes revisores em diferenciá-las das de outros artrópodes. Quanto a estes, foram positivas entre 10 e 15\% das caixas, anotando-se principalmente espécimes de baratas e aranhas seguidos de grilos e lagartas. № levantamento da rotina macro-regional do mesmo período, para 106.813 unidades domiciliares trabalhadas, capturaram-se 40.893 triatomíneos, em imensa maioria no âmbito peridomiciliar (91,6\%), correspondendo $92 \%$ ao Triatoma sordida, 5,6\% ao Panstrongylus megistus e 0,7\% ao Triatoma infestans, restando 1,6\% de o utras espécies ( T. pseudomaculata, T. arthurneivai, T. vitticeps, Rhodnius neglectus, P. diasi e P. geniculatus) .

Observa-se que foram apenas detectados raríssimos focos residuais de T. infestans, à época, em quatro dos doze municípios deste trabalho, correspondendo a cinco exemplares pela pesquisa rotineira ${ }^{11}$. Quanto ao custo estimado para os sete municípios aqui investigados na subárea " $\mathrm{A}$ " (quatro revisões de sensores), ao preço genérico de US\$1.50 por sensor e de US\$5.00 por unidade domiciliar visitada, 0 custo final de uma revisão sairia por ano a US\$5,598.00, sendo de US\$1,098.00 0 custo dos sensores (acréscimo de 14\% para reposição de sensores danificados) e US\$4,500.00 as quatro visitas realizadas ( seriam US\$2,223.00 para uma revisão única).

Já em termos do tempo de inspeção, indubitavelmente os sensores levam vantagem, demandando menos de quinze minutos por casa visitada (incluindo conversação) , contra a hora exigida na visita rotineira. Esta diferença, consequentemente, influirá no rendimento final da equipe de trabalho, pois na rotina vigente de inspeção - para a realidade atual de Minas Gerais - 0 rendimento oscila entre quatro e seis unidades/homem/dia, podendo esta média subir pelo menos 30 ou $40 \%$ no trabalho de revisão dos sensores.

Sobre a aceitação e a operacionalidade dos bio-sensores, de modo geral foram boas segundo os agentes da FUNASA e a população entrevistada a respeito, apenas sendo algo problemática a fixação das caixas às paredes da casa, 0 que demandou com frequêencia o uso de pregos. Segundo vários relatos de supervisão, a população aceitou bem os bio-sensores, que por suas figuras externas e seu aspecto agradável foram freqüentemente incorporados também como enfeites, pelos moradores. Não foram especificamente avaliadas as perdas de sensores, informando os agentes de saúde que, aparentemente, de modo geral foram retiradas por crianças e/ou despegaramse das paredes durante limpeza das mesmas.

\section{DISCUSSÃ0}

Em áreas chagásicas sob ação de controle químico adequado e continuado, é regra a imediata e progressiva redução dos triatomíneos domiciliados, fato observado desde 0 trabalho pioneiro de Emmanuel Dias e conforme a experiência acumulada até hoje ${ }^{35151629323336}$. Cabe, a seguir, permanente vigilância epidemiológica para controle de focos residuais e/ou adventícios ${ }^{562032}$. Embora possível, é onerosa e lenta a vigilância exercida em regime verticalizado, por equipes governamentais de saúde, sendo preferível em nosso país uma ação horizontalizada, de nível municipal, baseada em ampla participação comunitária 15162733 .

Em paralelo, a questão da baixa densidade triatomínica soe diminuir bastante a sensibilidade dos métodos de busca ativa, tipo "hora-homem", sendo altamente desejável a disponibilidade de técnicas e métodos de detecção mais simples, sensível e barata $^{2} 91023262822$.

Armadilhas têm sido experimentadas neste sentido, como as luminosas e aquelas que empregam atrativos vivos, e os chamados bio-sensores passivos para detecção de triatomíneos e/ou seus vestígios, como a caixa de Gómez-Nuñes, o sensor "Maria" e os calendários de Marsden, ensaiados desde os anos $1970^{81716182022273235}$.

De modo geral a eficácia destes instrumentos tem se mostrado relativamente discreta, em alguns trabalhos do Brasil e do Chile ${ }^{1322} 23$, assim como no Paraguai (A Rojas de Arias: comunicação pessoal, 1999). Não obstante, nos últimos lustros, estes sensores tiveram largo emprego e difusão na Argentina, com estímulo do TDRe adoção pelo Programa Nacional contra a doença de Chagas, chegando se à produção industrial em 199242131.

Maior entusiasmo sobre a ferramenta parece ter ocorrido ao início dos 1990, seguindo-se arrefecimento e menor uso em anos posteriores. Chamam particularmente atenção as menções a respeito feitas por dois recentes comitês de especialistas convocados pelo TDR/OMS sobre o controle da doença de Chagas, com intervalo de 11 anos (1989 e 2000): no primeiro, há indicação genérica do uso de sensores ( pág. 52) , o que não ocorre no segundo, que mais bem volta a enfatizar a necessidade de investigação e desenvolvimento de novas ferramentas para a detecção de triatomíneos em situações de baixa densidade (pág. 90) ${ }^{3637}$.

No Brasil, campo do interesse imediato do presente trabalho, o panorama epidemiológico da doença de Chagas tem evoluído e caminhado progressivamente para algumas situações pontuais no que toca à situação vetorial ${ }^{6719} 273334$ : a) redução drástica da infestação triatomínica intradomiciliar, raramente ultrapassando no País a média de 3\% dos focos detectados; b) Redução em geral da densidade triatomínicotripanosômica domiciliar nas áreas regularmente trabalhadas; c) predomínio absoluto de focos residuais e adventícios no âmbito peridomiciliar, mormente à custa de espécies secundárias e ubiquistas, e, d) virtual eliminação do T. infestans, remanescendo esta espécie em poucos focos residuais de baixa densidade em apenas dois Estados da Federação. Em termos de custo, apropriado o preço dos sensores utilizados no presente trabalho e sua revisão (US\$4,500.00, vide acima), esta estratégia resulta muito mais cara que US\$1,125.00 calculados para uma visita anual tipo homem-hora, conforme estimativas clássicas no Brasil e Argentina ${ }^{12} 2330$.

A ver pelos resultados acima, em que pesem algumas limitações metodológicas e operacionais, não parece restar dúvida que os bio-sensores "Maria" não mostraram a efetividade (por baixa sensibilidade) que deles se poderia esperar no presente ensaio, indicada por qualquer dos parâmetros 
classicamente utilizados ${ }^{73}$. Isto também fora observado por Garcia-Zapata e colaboradores em área de resíduo de T. sordida, em Goiás, após a eliminação de Triatoma infestans ${ }^{1516}$. No caso do presente trabalho, também não foi detectada a colonização dos sensores por T. sordida, também corroborando observações anteriores no Brasil que se mostraram claramente menos sensíveis em detectar a infestação das vivendas na região que a pesquisa direta por agentes s $^{170} 2425$.

Tendo atingido um grau de controle de triatomíneos intradomiciliados de boa qualidade, os desafios básicos ao programa de controle vetorial se voltam ao tripé peridomicílio, baixas densidades e espécies ubiquistas e secundárias, como T. sordida, T. brasiliensis, T. pseudomaculata ${ }^{356912151926 .}$ Os ditos sensores efetivamente não se adaptam ao âmbito peridomiciliar, face à múltiplas variedades de ecótopos existentes e à clara perspectiva de sua rápida deterioração quando expostos ao tempo $0^{4} 62635$. Além disto, também sua sensibilidade no intradomicílio mostrou-se menos efetiva que a metodologia de busca ativa direta, nas condições do presente ensaio. Outrossim, não há disponibilidade de informações da experiência argentina para espécies como 0 T. sordida e para situações peridomiciliares, que caracterizam as áreas aqui estudadas ${ }^{45}$, efetivamente não sendo comparáveis as duas realidades.

No presente ensaio, representativo de grande parte da área endêmica brasileira, o comportamento da espécie principal ( T. sordida) mostra-se muito constante, com invasões esporádicas do intradomicílio (onde os sensores atuam) e baixa capacidade de colonização dos mesmos ${ }^{1215192629}$. Para 0 Brasil, evidencia-se hoje a progressiva e adiantada fase de redução do T. infestans e de outras espécies intradomiciliares, restando em todas as áreas endêmicas os focos secundários e peridomiciliares de baixa densidade, sendo mais capturadas aquelas ubiquistas como T. sordida, T. brasiliensis, T. pseudomaculata, T. rubrovaria, T. vitticeps, P. megistus, R. neglectus e R nasutus ${ }^{91934}$. Com respeito à eficiência, apesar da extrema simplicidade do uso dos sensores e de seu maior rendimento em termos de casas revisadas/homem/dia, ainda se mostrou mais vantajosa a metodologia rotineira (especialmente a atual, descentralizada), conforme os valores apropriados em vários trabalhos ${ }^{12} 2630$.

Todos estes fatos, agregados a experiências como a presente, não indicam 0 emprego rotineiro de bio-sensores como 0 "Maria" nas atividades atuais de rotina de controle triatomínico, no Brasil, especialmente quando a maioria absoluta dos focos residuais de triatomíneos se encontra no peridomicílio 6834 . Resta lembrar que, em situação de vigilância, são fundamentais a educação sanitária e a participação comunitária, para que a população detecte diretamente eventuais triatomíneos e para manter a higiene da habitação e seus anexos, assim como para preservar instrumentos de detecção ${ }^{3616}{ }^{35}$. Concluindo, o presente ensaio ratifica trabalhos anteriores, igualmente numa área de Triatoma infestans (eliminado) e T. sordida (remanescente), mostrando a pouca eficiência dos sensores empregados, neste tipo de situação $0^{162022}$.

\section{REFERÊNCIAS BIBLIOGRÁFICAS}

1. Akhavan D. Análise de custo-efetividade do programa de controle da doença de Chagas no Brasil., Organização Panamericana da Saúde, Brasília, 1998.

2. Basombrio MA, Schofield CJ, Rojas CL, Del Rey EC. A cost-benefit analysis of Chagas Disease control in north-western Argentina. Transactions of the Royal Society of Tropical Medicine and Hygiene 92: 137-143, 1998.

3. Carlier Y, Dias JCP, Luquetti A0, Hontebeyrie H, Torrico F, Truyens C. Trypanosomiase americaine ou maladie de Chagas. Encyclopedíe MédicoChirurgicale. Paris, Editions Scientifiques et Medicales Elsevier, Maladies infectieuses 8 (A-20) : 1-21, 2002.

4. Chuit R. Control vectorial de la enfermedad de Chagas. In: Madoery RJ, Madoery C, Cámera MJ ( orgs) Actualizaciones en la Enfermedad de Chagas. Córdoba, Congreso Nacional de Medicina 265-268, 1993.

5. Dias E. Profilaxia da doença de Chagas. O Hospital 51: 485-498, 1957.

6. Dias, JCP. Chagas disease control in Brazil: which strategy after the attack phase? Annales de la Societé Bélgique de Medicine Tropicale 71 ( Supl 1) : 75-86, 1991.

7. Dias JCP. Controle. In: Dias JCP, Coura JR ( orgs.) Clínica e Terapêutica da Doença de Chagas. Uma abordagem prática para o clínico geral, Editora FIOCRUZ, Rio de Janeiro, p. 453-468, 1997.

8. Dias JCP. 0 controle da doença de Chagas no Brasil. In: Silveira AC ( org) El control de la enfermedad de Chagas em los Países del Cono Sur de América: historia de uma inciativa internacional 1991-2001. Brasília, Organização Panamericana da Saúde, p. 145-250, 2002.

9. Dias JCP, Schofield CJ. The evolution of Chagas Disease (American Trypanosomiasis) control after 90 years since Carlos Chagas discovery. Memórias do Instituto Oswaldo Cruz 94 (suppl 1):103-122, 1999.

10. Dias JCP, Silveira AC, Schofield CJ. The impact of Chagas Disease Control in Latin America - A review. Memórias do Instituto Oswaldo Cruz 97: 603-612, 2002.

11. Dias JCP, Vinhaes MC, Machado EMM, Azeredo BVM. Interrupção da transmissão vetorial da doença de Chagas por Triatoma infestans em Minas Gerais. Documento interno da Secretaria de Estado da Saúde de Minas Gerais e da FUNASA/MG para instrução da Comissão Internacional de Avaliação da Iniciativa do Cone Sul da OPAS/OMS, reprografado, Belo Horizonte, 2001.

12. Diotaiuti LG, Paula OR, Falcão PL, Dias JCP. Evaluation of the Chagas Disease vector control programme in Minas Gerais, Brazil, with special reference to Triatoma sordida. Bulletin of the Pan American Health Organization 28: 211-219, 1994.

13. Forattini OP, Juarez E, Correia RR. Medida da infestação domiciliar por Triatoma infestans. Revista de Saúde Pública 3: 11-16, 1969.

14. Fundação Nacional de Saúde. Normas Técnicas para o Controle da Doença de Chagas. Ministério da Saúde, Brasília, 1996.

15. Garcia-Zapata MTA. Vigilância no controle da doença de Chagas em duas áreas endêmicas de Triatoma infestans. Tese de Mestrado, Universidade de Brasília. Brasília, DF, 1985.

16. Garcia-Zapata MTA, Schofield CJ, Marsden PD. A simple method for detecting the presence of live triatomine bugs in houses sprayed with insecticides. Transactions of the Royal Society of Tropical Medicine and Hygiene 79: 5458-5459, 1985.

17. Garcia-Zapata MTA, Marsden PD. Enfermedad de Chagas: control y vigilancia con insecticidas y participación comunitaria en Mambaí, Goiás, Brasil. Boletín de la Oficina Sanitaria Panamericana 116: 97-110, 1994.

18. Gomez-Nuñes JC. Desarrollo de un nuevo método para evaluar la infestación intradomiciliaria por Rhodnius prolixus. Acta Científica Venezolana, 16: 26-31, 1965.

19. Lederbour CC, Dias JCP, Vinhaes MC. Relatório Final. II Reunião anual de avaliação do programa de controle da doença de Chagas no Brasil (Uberaba, mg, 22 a 24 de outubro de 2003). Brasília, Boletim Eletrônico da Secretaria de Vigilância em Saúde, Departamento de Vigilância Epidemiológica, Coordenação Geral de Doenças Transmissíveis, 2004. 
20. Marsden PD, Penna RA. Vigilance unit for household subjects to triatomine control. Transactions of the Royal Society of Tropical Medicine and Hygiene 76: 790-792, 1982.

21. Moncayo A. Progress towards interruption of transmission of Chagas Disease. Memórias do Instituto Oswaldo Cruz 94 ( $\sup 1$ ) : 401-404, 1999.

22. Noireau F, Flores R, Vargas F. Trapping sylvatic Triatominae (Reduviidae) in hollow trees. Transactions of the Royal Society of Tropical Medicine and Hygiene 93: 13-14, 1999.

23. Oliveira Filho AM. Cost-effectiveness analysis in Chagas' disease vector's control interventions. memórias do Instituto Oswaldo Cruz 84 ( supl IV): 409-417, 1989.

24. Oliveira Filho AM. Uso de nuevas herramientas para el control de triatominos em diferentes situaciones entomológicas em el continente americano. Revista da Sociedade Brasileira de Medicina Tropical 30: 41-46, 1997.

25. Pinchin R, Fanara DM, Castleton CW, Oliveira Filho AM. A comparative study of domestic survey techniques for the Chagas Disease vector Triatoma infestans. Insect Science and Application 3: 79-84, 1982.

26. Rodrigues VLCC, Ferraz Filho NA, Silva EOR, Lima VLC. Prevalência, índices de infecção e hábitos alimentares de triatomíneos capturados em uma área de vigilânvia entomológica. Revista da Sociedade Brasileira de Medicina Tropical 25: 183-190, 1992.

27. Schmunis GA, Dias JCP. La reforma del sector salud, descentralización, prevención y control de enfermedades transmitidas por vectores. Cadernos de Saúde Pública 16 ( supl 2) : 117-123, 2000.

28. Schofield CJ. A comparison of sampling techniques for domestic populations of Triatominae. Transactions of the Royal Society of Tropical Medicine and Hygiene 72: 449-455, 1978.
29. Schofield CJ. Triatominae: biologia y control. Eurocommunica Publications, London, 1994.

30. Schofield CJ, Dias JCP. A cost-benefit analysis of Chagas Disease control in Brazil. Memórias do Instituto Oswaldo Cruz 86: 285-295, 1991.

31. Segura EL. El control de la enfermedad de Chagas em la República Argentina. In Silveira AC ( org.) El control de la enfermedad de Chagas em los Países del Cono Sur de América: historia de uma inciativa internacional 1991-2001. Organização Panamericana da Saúde, Brasília, p. 45-108, 2002.

32. Silva EOR. Profilaxia. In: Brener Z, Andrade ZA (orgs) Trypanosoma cruzi e doença de Chagas. Guanabara Koogan, Rio de Janeiro, p. 425-449, 1979.

33. Silveira AC. Profilaxia. In: Brener Z, Andrade ZA, Barral Neto M (orgs) Trypanosoma cruzi e doença de Chagas (2ª Edição). Rio de Janeiro, Guanabara Koogan Editora, p. 75-87, 1999

34. Vinhaes MC, Dias JCP. Doença de Chagas no Brasil. Cadernos de Saúde Pública 16 ( supl 2) : 7-12, 2000.

35. Wisnivesky-Colli C, Paulone I, Pérez A, Chuit R, Gualtieri JM, Solarz N, Smith A, Segura EL. A new tool for continuous detection of the presence of triatomine bugs, vectors of Chagas' Disease, in rural households. Medicina (Buenos Aires) 47: 45-50, 1987.

36. World Health Organization. Control of Chagas Disease. WHO Technical Report Series 811, World Health Organization, Geneva, 1991.

37. World Health Organization. Control of Chagas Disease. WHO Technical Report Series 905, World Health Organization, Geneva, 2002. 